\title{
Spectrum Sensing in Cognitive Radio by Use of Volume-Based Method
}

\author{
C. S. Preetham ${ }^{1}$, Ch. Mahesh ${ }^{2}$, Ch. Saranga Haripriya ${ }^{2}$, Ramaraju Anirudh ${ }^{2}$, M. S. Sireesha ${ }^{2}$ \\ ${ }^{1}$ Assistant Professor, Department of ECE, Koneru lakshmaiah Education Foundation, Andhra Pradesh, India \\ ${ }^{2}$ U G Student, Department of ECE, Koneru lakshmaiah Education Foundation, Andhra Pradesh, India \\ *Email: ramaraju.anirudh@gmail.com
}

\begin{abstract}
Spectrum sensing is the mission of finding the licensed user signal situation, i.e. to determine the existence and deficiency of primary (licensed) user signal, the recent publications random matrix theory algorithms performs better-quality in spectrum sensing. The RMT fundamental nature is to make use of the distributed extremal eigenvalues of the arrived signal sample covariance matrix (SMC), specifically, Tracy-Widom (TW) distribution which is useful to certain extent in spectrum sensing but demanding for numerical evaluations because there is absence of closed-form expression in it. The sample covariance matrix determinant is designed for two novel volume-based detectors or signal existence and deficiency cases are differentiated by using volume. Under the Gaussian noise postulation one of the detectors theoretical decision thresholds is perfectly calculated by using Random matrix theory. The volume-based detectors efficiency is shown in simulation results.
\end{abstract}

Keywords: Cognitive Radio, Spectrum sensing, Energy Detector, Volume-Based Method, Licensed user, Unlicensed user

\section{Introduction}

It has been exposed that the existing policy of fixed spectrum distribution do not fully consume the offered spectrum cognitive radio[1-4], whose main theme is to sense the spectrum over a wide range of frequency band and make use of temporally unoccupied bands for opportunistic wireless transmission ,is a promising pattern to increase the spectrum usage efficiency. In a cognitive radio network, when the spectrum property of a primary user are not occupied, a secondary user is allowed to use them. That is to tell, the secondary user needs to consistently sense the presence of the primary user. This is referred to as spectrum sensing, which can be cast as a binary hypothesis testing problem and is predominantly challenging for small sample size and/or low signal to -noise ratio(SNR) conditions.

The data which consist of noise and are can be implicit to be independent and identically distributed (IID) for the information of signal-absence case. But in the case of Primary User signal is present it can be apparent that the energy and the observations of the correlation structures are diverge. By use of these dissimilarities we can achieve the spectrum sensing as a result. The optimal result for the IID PU signals has been revealed by the energy detector ED[5] when the noise power is recognized. Its estimate is used alternative [6]. When the noise power in sequence is frequently occupied in observe. When it is moderately perceptive of the noise, the detection concert of the energy detection will be reduce. In the case of noise only present which is comparable to the scaled identity matrix in the asymptotic sense is less consequential Eigen values then the Eigen values of the received signal sample covariance matrix in the signal presence. The results of Eigen spectrum are from the correlation structure in the covariance matrix. In the literature [613] the correlation structure for spectrum sensing have been exploit by the number of Eigen value based detectors. The correlated signals embedded in the IID noise can be recognize by the Arithmetic to Geometric Mean (AGM) method is consequent in the framework of Generalized Likelihood Ratio Test (GLRT). The theoretical threshold cannot be determined precisely when the temporal and spatial dimensions are incompetent because of AGM algorithm has its root in the maximum likelihood theory which turns out to be insufficient. On the other hand the SCM resembles to an identity matrix or its correlated with the use of maximum and minimum Eigen values in the Maximum to Minimum Eigen (MME) approach is advanced. For the feeble correlated signals or/and small sizes is detection performance is highly sensitive only when Eigen values are not consumed. 


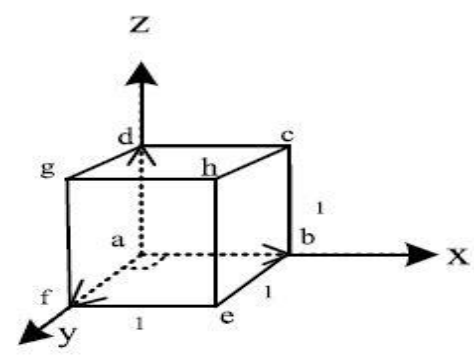

(a) $v_{1}=1$

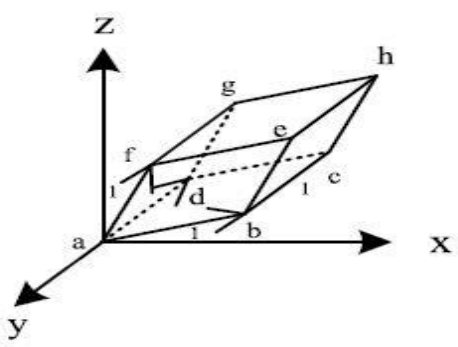

(b) $\mathrm{v}_{2}<\mathrm{v}_{1}$

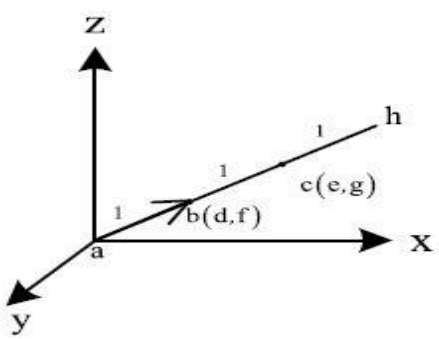

(c) $\mathrm{v}_{3}=0$

Fig.1. Volume comparison for signal absence and signal presence cases.

The spreading of the maximum and minimum Eigen values which are used in the framework of the random matrix theory (RMT), namely, the Tray widow (TW) distribution [14] is relies to calculate the theoretical threshold values for MME algorithm. Then the additional overhead of numerical evaluation is required when there is an indication of explicit closed from expression for the TW distribution. An exact variant of the GLRT has been devised for the spectrum sensing when there is only single primary signal in[12] which is equivalent to the signal to noise Eigen value method[10].The number of primary signals in the sensed GLRT test[15],

Hadamard ratio test[16]to handle the no uniform noise. In this work, the detection of primary users in attractive manner, a new philosophy is formulated for spectrum sensing. In the signal presence and signal absence situations are for the determinant of SCM or volume differ.

The respite of the paper is as follows . The problem formulation of spectrum sensing is presented in section2.In section 3, prior to derive the volume based detectors. The impulse is provided via geometric interpretation. Then two volume based detectors, denoted by VD1, and VD2 are urbanized for spectrum sensing, with the use of RMT, the theoretical decision threshold of the VD2 is truthfully determined and no numerical procedure is involved. Simulation results are included in section4 to estimate the performance of the proposed detectors by comparing with the ED, AGM, MME, Hadamard ratio and SNE methods.

\section{Problem Formulation}

Let $\mathrm{d}$ be the number of users in a multipath fading environment such that $d \geq 1$. We consider (d-1) secondary users and 1 primary user. The SU's and PU's operate with single antenna. The presentation can be further simplified by taking the available secondary users also as primary users, so now the number of primary users are ' $d$ '. To monitor the channel a secondary user with $\mathrm{m}$ antennas is needed.

The output of secondary user is represented with $y_{i}(k)$, where the limits of independent observations is $\mathrm{i}=1$ to $\mathrm{m}$ and $\mathrm{k}=1$ to $\mathrm{n}$.

$$
\begin{aligned}
& y(k)=w(k), \quad H_{0} \\
& H S(k)+w(k), \quad H_{1} .
\end{aligned}
$$

Where $H_{0}$ and $H_{1}$ are the signal present and signal absence cases

$$
\begin{gathered}
y(k)=\left[x_{1}(k) \ldots . . x_{m}(k)\right] \\
s(k)=\left[s_{1}(k), \ldots ., s_{d}(k)\right]
\end{gathered}
$$

$$
w(k)=\left[w_{1}(k), \ldots \ldots, w_{m}(k)\right] .
$$

We should have to consider the channels i.e. primary signals and noise are considered to be real Value throughout this paper if $H \notin$ $R^{m \times d}$ so $\in R^{m \times d}$. When we considering the primary users and secondary users $H \in R^{m \times d}$ represents the fading channels between the primary user and secondary user. $H \in R^{m \times d}$ Stands for observation, signal and noise vector respectively. So we have to imagine the noises are statistically independent and we have the equation $w_{i}(k) \sim N\left(0, \sigma_{w_{i}}^{2}\right) \quad(\mathrm{i}=1$ to $\mathrm{m})$. Where , $\sigma_{w_{i}}^{2}$ is the unknown noise variance, $\sim$ represents "distribute-d as" and $(\mu, \Sigma)$ represents the Gaussian distribution with mean $\mu$ and variance $\boldsymbol{\Sigma}$. if $\sigma_{w_{i}}^{2}=\sigma_{w}^{2}$ for $\mathrm{i}=1$ to $\mathrm{m}$ the noise becomes Independent and Identically Distributed (uniform);otherwise, it is the non-uniform noise due the uncalibrated receiver[15,17]. In the mean while we can consider the random process $s_{i}(k)(i=1, \ldots, d)$ this $s_{i}(k)$ is having mean zero and unknown variance $\sigma_{s_{i}}^{2}$, which is independent of noise. Note that the primary signal vector $\mathrm{s}(\mathrm{k})$ is unnecessarily Gaussian distributed. We employ the covariance matrix of $\mathrm{y}(\mathrm{k})$,In order to exploit the correlation structure in the observations and that covariance matrix is

$$
R=E\left[y(k) y^{T}(k)\right]
$$

Where $\mathrm{E}[$.$] is the expectation operator.$

\section{Volume-based detector for spectrum sensing}

\subsection{Statistical Elucidation:}

The hyper-volume of the Geometry resolute by the row vectors of R, is the determinant of $\mathrm{R}$. As an example, let us think about the situation of three receiving antennas where the pragmatic with unity variance and Null mean may be correlated or coherent and independent. It says that the resultant covariance matrices are the 3X3 identity matrix, full-rank non solidary matrix and rank-one arbitrary matrix. In fig 1 , it depicts that the geometrics specifies cube, parallelepiped and line, shaped by the row vectors of the matrices. In fig 1 all the edges of the geometries are assumed to be unity such that $\|\mathrm{R}(\mathrm{i},:)\|=1$ with $\mathrm{R}(\mathrm{i},:$ ) $)$ being the $i^{\text {th }}$ row of $\mathrm{R}$ and $\|$. $\|$ being the Euclidean norm. Let $v_{1}, v_{2}$, and $v_{3}$ denotes the volume of the cube, parallelepiped and line respectively. The cube match up with the case of signal absence whereas the other two geometries that is, parallelepiped and line referring to the signal existence case. The covariance matrix is a $3 \mathrm{X} 3$ identity matrix for the situation of signal deficiency, that is $\mathrm{R}=I_{3}$, whose rows determine the coordinates of the points $b$, $f$ and $d$ in fig 1(a), that is that is, 
$\left(x_{b}, y_{b}, z_{b}\right)=(1,0,0),\left(x_{f}, y_{f}, z_{f}\right)=(0,1,0), \quad\left(x_{d}, y_{d}, z_{d}\right)=$ $(0,0,1)$. Accordingly, we obtain $v_{1}=1$. For the signal existence case the diagonal matrix arrangement is damaged, leading to volume reduction, as indicated in fig $1(\mathrm{~b})$ and $1(\mathrm{c})$. To differentiate the Licensed user from background noise we develop a new methodology for accurate spectrum sensing i.e; volume based method. In this volume is differentiating the licensed users from background noise.

\subsection{Derivation:}

Let us consider when there is no signal, the independent observations of $y_{i}(k), \mathrm{i}=1$ to $\mathrm{m}$, and $\mathrm{k}=1$ to $\mathrm{n}$ which are formed by the elements of secondary user output $\mathrm{y}(\mathrm{k})$. By evaluating the sample covariance matrix, instead of population covariance matrix $R$, we develop the correlation structure for spectrum sensing. The SCM is given by

$$
\mathrm{S}=\frac{1}{\mathrm{n}} \sum_{\mathrm{k}=1}^{\mathrm{n}} \mathrm{y}(\mathrm{k}) \mathrm{y}^{\mathrm{T}}(\mathrm{k})
$$

In the meantime by using $\delta_{i}=\|\mathrm{S}(\mathrm{i},:)\|(\mathrm{i}=1$ to $\mathrm{m})$ the edge lengths of covariance matrix which are incorporated with row vectors are calculated. The $\operatorname{det}\left[\mathrm{D}^{-1} \mathrm{~S}\right]$ which is the volume of the geometry with solidarity is obtained by $\mathrm{D}=\operatorname{diag}\left(\delta_{1}\right.$ to $\left.\delta_{m}\right)$. Apply logarithm we have

$$
\varepsilon_{1} \triangleq \operatorname{logdet}\left[\mathrm{D}^{-1} \mathrm{~S}\right]
$$

For the situation of signal nonappearance as the number of samples tends to endlessness the $\mathrm{D}^{-1} \mathrm{~S}$ should be identity matrix, which is prompting to the volume of one. For the circumstance of signal appearance, in any case, the impressive lessening of volume obtained by the connection between the rows of $\mathrm{D}^{-1} \mathrm{~S}$, giving a decent sign to the primary signal. In this manner, contrasted and a foreordained limit $\gamma_{1}$, the statistic $\varepsilon_{1}$ can yield revise location of the primary users. That is

$$
\varepsilon_{1}^{\stackrel{\mathrm{H}_{0}}{>} \underset{\mathrm{H}_{1}}{>}} \gamma_{1}
$$

Be that as it may, as the circulation of $\operatorname{det}\left[\mathrm{D}^{-1} \mathrm{~S}\right]$ is obscure, it is difficult to decide the hypothetical edge for $\varepsilon_{1}$. To reduce the trouble, we accept that the noise is independent and identically distributed and determining the proportionate measurement. It is anything that difficult to get

$$
\begin{aligned}
\log \operatorname{det}\left[\mathrm{nD}^{-1} \mathrm{~S}\right] & =\operatorname{logdet}\left[\widehat{\sigma}_{\mathrm{w}}^{2} \mathrm{D}^{-1} \times \frac{\sigma_{\mathrm{w}}^{2}}{\widehat{\sigma}_{\mathrm{w}}^{2}} \times \frac{\mathrm{nS}}{\sigma_{\mathrm{w}}^{2}}\right] \\
& =\operatorname{logdet}\left[\frac{\mathrm{nS}}{\sigma_{\mathrm{w}}^{2}}\right]-\log \operatorname{det}\left[\frac{\mathrm{D}}{\hat{\sigma}_{\mathrm{w}}^{2}}\right]-\operatorname{mlog}\left(\frac{\widehat{\sigma}_{\mathrm{w}}^{2}}{\sigma_{\mathrm{w}}^{2}}\right) .
\end{aligned}
$$

The estimated noise variance $\widehat{\sigma}_{\mathrm{w}}^{2}$ is computed by $\widehat{\sigma}_{\mathrm{w}}^{2}=\operatorname{var}(\mathrm{Y}(:))$. In this $\mathrm{y}(:)$ is comes about because of the vectorization of $\mathrm{Y} \triangleq$ $[y(1), \ldots ., y(n)]$. Therefore we get,

$$
\begin{aligned}
\varepsilon_{2} & \triangleq \log \operatorname{det}\left[\frac{\mathrm{nS}}{\sigma_{\mathrm{w}}^{2}}\right] \\
& =\log \operatorname{det}\left[\mathrm{nD}^{-1} \mathrm{~S}\right]+\operatorname{logdet}\left[\frac{\mathrm{D}}{\hat{\sigma}_{\mathrm{w}}^{2}}\right]+\operatorname{mlog}\left(\frac{\widehat{\sigma}_{\mathrm{w}}^{2}}{\sigma_{\mathrm{w}}^{2}}\right) .
\end{aligned}
$$

It ought to be noted that, for the hypothesis $\mathrm{H}_{0}$ which is obtained in noise only case, empowering us to precisely decide the limit for $\varepsilon_{2}$ gave that the keep going term on the RHS of (10) can be fixed. For the hypothesis of $\mathrm{H}_{1}$ which is obtained in signal appearance case, $\widehat{\sigma}_{\mathrm{w}}^{2}$ can't be effectively evaluated, making $\varepsilon_{2} / \mathrm{H}_{1}$ to have a alternate conduct from $\varepsilon_{2} / \mathrm{H}_{0}$ and there by empowering us to accurately recognize the presence of the primary signal. The term $\operatorname{mlog}\left(\frac{\widehat{\sigma}_{\mathrm{w}}^{2}}{\sigma_{\mathrm{w}}^{2}}\right)$ can't be figured from $\mathrm{y}(\mathrm{k})$. It should be calculated from $\sigma_{\mathrm{w}}^{2}$ is the true noise variance. To dodge this issue, we infer a gauge to inexact it. To this end, setting

$$
\widehat{b}=\operatorname{mlog}\left(\frac{\widehat{\sigma}_{\mathrm{w}}^{2}}{\sigma_{\mathrm{w}}^{2}}\right) .
$$

And in the noise only case the noise variance can be precisely figured from $m n \times 1$ perceptions $y(:)$, we use $E[b]$ approximate $\hat{b}$, or

$$
\mathrm{E}[\hat{\mathrm{b}}] \approx \hat{\mathrm{b}} \text {. }
$$

If $\hat{b}$ is much littler than the other two terms in (10) the above approximation is substantial. Then again, it takes after from [18] that

$$
\frac{\widehat{\sigma}_{\mathrm{w}}^{2}}{\sigma_{\mathrm{w}}^{2}} \triangleq \frac{1}{\mathrm{mn}} \mathrm{u} \sim \frac{1}{\mathrm{mn}} \chi 2(\mathrm{mn}) .
$$

Where $\mathrm{u} \sim \chi 2(\mathrm{mn})$ with $\chi 2(\mathrm{mn})$ being the chi-squared dispersion with $\mathrm{mn}$ degrees of opportunity substituting (13) into (11) and taking desire yield,

$$
\mathrm{E}[\hat{\mathrm{b}}]=\mathrm{mE}\left[\log \left(\widehat{\sigma}_{\mathrm{w}}^{2} / \sigma_{\mathrm{w}}^{2}\right)\right]=\mathrm{m}\left(\log \frac{1}{\mathrm{mn}}+\mathrm{E}[\log \mathrm{u}]\right) .
$$

We require the accompanying lemma [19] for continuing the above expression.

Lemma 1. If $\mathrm{u} \sim \chi 2(\mathrm{j})$, then

$$
E\left[\log u=\log (j)-\frac{1}{j}-\frac{1}{3 j^{2}}+\frac{2}{15 j^{4}}+O\left(j^{-6}\right)\right.
$$

Proof: The proof of lemma 1 is given in [19].

Therefore substituting (15) into (4) gives

$$
\begin{aligned}
E[\hat{b}] & =-m\left(\frac{1}{m n}+\frac{1}{3(m n)^{2}}-\frac{2}{15(m n)^{4}}-O\left(j^{-6}\right)\right) \\
& \approx-m\left(\frac{1}{m n}+\frac{1}{3(m n)^{2}}-\frac{2}{15(m n)^{4}}\right) \triangleq b .
\end{aligned}
$$

It takes after from (12) and (16) that $\hat{b}$ can be approximated by $\mathrm{b}$. Subsequently, the test measurement $\varepsilon_{2}$ in (10) can be re communicated as

$$
\varepsilon_{2}=\operatorname{logdet}\left[\mathrm{nD}^{-1} \mathrm{~S}\right]+\log \operatorname{det}\left[\frac{\mathrm{D}}{\hat{\sigma}_{\mathrm{w}}^{2}}\right]+b
$$

It ought to be brought up that, the principal term on the RHS much bigger than the other two terms. Due to this the fact that $\varepsilon_{2}$ depends on the $\widehat{\sigma}_{\mathrm{w}}^{2}$ which is evaluated noise variance, however, it is insensitive to the instability in the later. This can likewise be checked by the simulation results in section 4 . For the instance of signal-presence, be that as it may, the measured variable must have a alternate behavior because of the way that the volume in both 
hypothesis are distinctive. Accordingly, for a given threshold $\gamma_{2}$, the choice issue can be expressed as

$$
\varepsilon_{2} \stackrel{\mathrm{H}_{0}}{\underset{\mathrm{H}}{>} \gamma_{2}}
$$

The above derivations give the statistic results $\varepsilon_{1}$ and $\varepsilon_{2}$ for the proposed algorithms i.e. volume-based algorithm VD1 and VD2 respectively in spectrum sensing. Monte Carlo simulation gives the thresholds for both volume-based detection i.e. VD1 and VD2. In the subsequent subsection asymptotic theoretical threshold calculation for second volume-based detection VD2 is declared.

\subsection{Asymptotic theoretical threshold for vd2}

The detection probability and false alarm probability i.e. $\mathrm{P}_{\mathrm{d}}$ and $\mathrm{P}_{\mathrm{fa}}$ respectively are calculated in below. The probability density function (PDFs) in the hypotheses $\mathrm{A}_{0}$ and $\mathrm{A}_{1}$ of $\varepsilon_{2}$ are denoted as $\mathrm{G}_{0}(\mathrm{t})$ and $\mathrm{G}_{1}(\mathrm{t})$ respectively.

$$
\begin{aligned}
& P_{f a}=\operatorname{Prob}\left(\varepsilon_{2}<\gamma_{2} \mid A_{0}\right)=\int_{-\infty}^{\gamma_{2}} f_{0}(t) d t \\
& P_{d}=\operatorname{Prob}\left(\varepsilon_{2}<\gamma_{2} \mid A_{1}\right)=\int_{-\infty}^{\gamma_{2}} f_{1}(t) d t
\end{aligned}
$$

Based on the different environmental conditions the statistic of the received signal varies which leads difficulty in the evaluation of $\mathrm{f}_{1}(\mathrm{t})$, although the results of detection probability $\left(\mathrm{P}_{\mathrm{d}}\right)$ and false alarm probability $\left(\mathrm{P}_{\mathrm{fa}}\right)$ gives $\mathrm{f}_{0}(\mathrm{t})$ and $\mathrm{f}_{1}(\mathrm{t})$.

On the other hand by using Jonsson theorem $f_{0}(t)$ is resoluted with no trouble.

\section{Theorem 1:}

In this theorem the variables with unity variance and zero mean i.e. $\mathrm{y}_{\mathrm{i}}(\mathrm{k}), \mathrm{i}=1, \ldots ., \mathrm{m}$, where $\mathrm{k}=1,2, \ldots \ldots, \mathrm{n}$, are self-determining variables. If the value of $\mathrm{c}$ is considered as a constant when $\mathrm{m}, \mathrm{n} \rightarrow \infty$ and $\mathrm{m} / \mathrm{n} \rightarrow$ $c \in(0,1)$ then $\varepsilon_{2}$ is Gaussian distributed.

$$
\varepsilon_{2}=\log \operatorname{det}\left(\begin{array}{c}
n s \\
\sigma_{w}^{2}
\end{array}\right) \sim N\left(\mu_{\varepsilon}, \sigma_{w}^{2}\right)
$$

Where $\sigma_{\varepsilon}^{2}=-2 \log (1-\mathrm{c})$

$$
\mu_{\varepsilon}=\log (\mathrm{n}-1)_{\mathrm{m}} \text { and }(\mathrm{n}-1)_{\mathrm{m}}=(\mathrm{n}-1)(\mathrm{n}-2) \ldots \ldots(\mathrm{n}-\mathrm{m}) .
$$

Proof: Complete proof of theorem 1 is available in [19]

Equation 19 says in noise environment $\varepsilon_{2} \sim N\left(\mu_{\varepsilon}, \sigma_{w}^{2}\right)$

$$
\mathrm{P}_{\mathrm{fa}}=1-\int_{\frac{\gamma_{2}-\mu_{\varepsilon}}{\sigma_{\varepsilon}}}^{\infty} g(t) d t=1-\frac{\gamma_{2}-\mu_{\varepsilon}}{\sigma_{\varepsilon}}
$$

Now we can calculate the threshold $\gamma_{2}$ for false alarm level $\varepsilon=P_{f a}$ because $\mathrm{Q}(\mathrm{x})=\int_{x}^{\infty} g(t) d t$ by means of $\mathrm{g}(\mathrm{t})=1 / \sqrt{2 \pi} \exp \left(-t^{2} / 2\right)$.

$$
\gamma_{2}=\sigma_{\varepsilon} Q^{-1}(1-\varepsilon)+\mu_{\varepsilon}
$$

$\gamma_{2}$ Is intended through $\mathrm{m} / \mathrm{n}$ where the constant value is replaced by $c=\log _{m, n \rightarrow \infty} m / n$ by using suggestions given by Bai and Silverstein from reference [21].

\section{Simulation Results}

\subsection{Accuracy of theoretical decision threshold}

Now, first evaluate the exact of the theoretical decision thresholds. For calculating theoretical thresholds of $\mathrm{VD} 1, \mathrm{VD} 2, \operatorname{ED}\left(\sigma_{w}^{2}\right) \operatorname{and} \operatorname{ED}\left(\hat{\sigma}_{w}^{2}\right)$ stands for the Energy Detection methods using to calculate the true and estimated noise variances .Meanwhile, the providing methods are associated for the test statistics. In this method, the Energy detection of the threshold value $\bar{\Gamma}^{-1}$ is the inverse of the incomplete gamma function as gammaincinv(.,.) in MATLAB. The inverse of the cumulative distribution function(CDF)of the TW distribution is denoted as $T_{1}^{-1}($.$) of order one { }^{2}[14]$ and then the inverse of the CDF of the $\mathrm{F}$ distribution[22] is denoted as $F^{-1}(., .,$.$) .Hence some number of state-$ of-the-art methods are considered, such as Roy's largest root test and some classical detectors and wilks' likelihood ratio test [23], which are not included.

According to the some given false alarm level, we have carried out 50,000 independent Monte Carlo trails in the absence of Primary users and select the decision threshold for investigated methods to exactly determine the simulated threshold. For $m=6$ and $n=12$ the numerical values are noted in Table 4, where $\triangleq\left(\mid \gamma_{\text {the }}\right.$ $\left.\gamma_{\text {sim }} \mid\right) / \gamma_{\text {sim }} \times 100 \%$ is calculated as Error with the theoretical and simulated thresholds as $\gamma_{\text {the }}$ and $\gamma_{\text {sim }}$. In this the noise type is IID (Independent and Identically Distributed) Gaussian process with null mean and unknown variance $\sigma_{w}^{2}$. For enabling the $\operatorname{ED}\left(\hat{\sigma}_{w}^{2}\right)$ method to work properly,we have to assume that there are $\mathrm{L}$ number of signal free samples are available for the noise variance.It is seen that when compared to the other algorithm the error of VD2 is more close to that of the $\operatorname{ED}\left(\sigma_{w}^{2}\right)$ algorithm. In fact, for the spectrum sensing the $\mathrm{ED}\left(\sigma_{w}^{2}\right)$ method cannot be employed as true noise variance is unknown to the receiver. Therefore, it is used as a benchmark. The exact theoretical threshold of the $\operatorname{ED}\left(\hat{\sigma}_{w}^{2}\right)$ can be determined in Table 4 by $\mathrm{F}$ distributed which is also in [24,25].For $\mathrm{n}=100$ the exact methods of theoretical threshold are in Table 5 .

\subsection{Detection performance}

By using the simulated threshold we can calculate the detection performance of Volume-based method and energy detection method. And the simulated threshold of proposed algorithm is also obtained by using 50,000 Monte Carlo trials which are independent, and other techniques thresholds are simulated in the tables mentioned in this section. The values of these tables are presented for the comparison of mentioned schemes. The output channel $\mathrm{H}$ which is obtained with zero mean Gaussian distribution is drawn randomly by distribution and again fixed for the duration of spectrum sensing. The SNR is evaluated using $10 \log _{10} \frac{\operatorname{tr}\left[R_{S}\right] / d}{\operatorname{tr}\left[R_{w}\right] / m}$ with $R_{S}=E\left[s(k) s^{T}(k)\right]$ and $R_{w}=E\left[w(k) w^{T}(k)\right]$ which makes the columns of output $\mathrm{H}$ to unity.

\subsection{Extension to complex-valued case}

$X(k)=X_{R}(k)+j X_{i}(k)$ is the representation of independent complex Gaussian function for noise assumption and $X_{R}$ and $X_{I}$ are real and imaginary variables, and $\mathrm{j}$ is represented as $j=\sqrt{-1}$. If real part $\mathrm{X}_{\mathrm{R}}(\mathrm{k})$ is correlated with imaginary part $\mathrm{X}_{\mathrm{I}}(\mathrm{k})$ then we will get $E\left[X(k) X^{H}(k)\right]=E\left[X_{R}(k) X_{R}^{T}(k)\right]+E\left[X_{I}(k) X_{I}^{T}(k)\right]=$ $E\left[y(k) y^{T}(k)\right]$ 
Where $y(k) \triangleq\left[X_{R}(k) X_{I}(k)\right]$.

A singular covariance matrix on behalf of $n<2 m$ is 1 formed when we observe the transform of complex to real corresponding item. If we perform this transformation then it is somewhat easy for the complex valued case in proposed method.

Basically $S_{i}(k)$ is a primary signal where $\mathrm{i}=1,2, \ldots, \mathrm{d}$. Let us believe that $S_{i}(k)$ is QPSK modulated with IID random symbols with values ${ }_{-}^{+} \sqrt{2} / 2{ }_{-}^{+} j \sqrt{2} / 2$ with the same probabilities. The MIMO Rayleighfading channel which are accurately illustrate the practical channels are studied in this paper. The $\sum$. with $(\mathrm{k}, 1)^{\text {th }}$ entry is determined as

$\sum_{k l}=\frac{I_{0}\left(\sqrt{k^{2}-4 \pi^{2} d_{k l}^{2}+j 4 \pi k \sin (\mu) d_{k l}}\right.}{I_{0}(k)}$

Where $(\mathrm{k}, \mathrm{l}=1,2, \ldots \mathrm{m})$

The width of the angles-of-arrival (AOAs) is controlled by $\mathrm{K}$ of the primary signals which are interrupting upon the antennas of secondary user. Signals are varies from 0 to $\infty$ where 0 represents the isotropic scattering and $\infty$ represents the non isotropic scattering. The direction of angle of arrivals are denoted as $\mu \in(-\pi, \pi)$, and the distance is denoted as $d_{k l}$, it is normalized with regards to the wavelength $\lambda$ involving $\mathrm{k}^{\text {th }}$ and $\mathrm{l}^{\text {th }}$ antennas of the unlicensed users. The modified Bessel function zeroth-order is $I_{0}($.). We set the values as $\mu=\pi / 2$ and $k=80$.The linear uniform array structure with the inter element distance of $\lambda / 2$ of the antennas of secondary user are to be assumed. And the distance between the adjacent antennas is 0.5

.Now we have to concentrate the behavior of the different detectors in the uncalibrated receiver. Now we consider the Quadrature phase shift keying (QPSK) signal which is the waveform of the solitary primary user. By this QPSK signal we concentrate the impact of non-uniform noise.

The figure 2 represents the performance of the volume based method in both uniform and non uniform noise cases for $\mathrm{m}=4$.

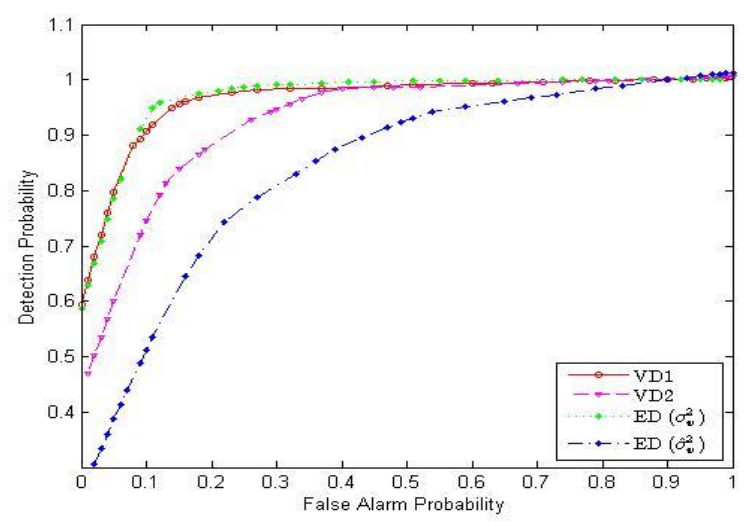

Fig.2(a) Uniform noise for $m=4$.

Fig. 2(a) Represents the ROC (Receiver operating Characteristics) of uniform noise (Independent and identically distributed) with Signal to Noise Ratio(SNR) is $-5 \mathrm{~dB}$, number of antennas(m) is 4 and the number of samples(n) is equals to 30 . The above figure says that, as compared with volume-based detector $2\left(V D_{2}\right)$ approach, the volume-based detector $1\left(V D_{1}\right)$ approach is very close to benchmark. The ED $\left(\hat{\sigma}_{w}^{2}\right)$ approach is basically overlooks the way that the noises are Independent and identically distributed. It is substandard as compared with $V D_{1}$ and $V D_{2}$ method.

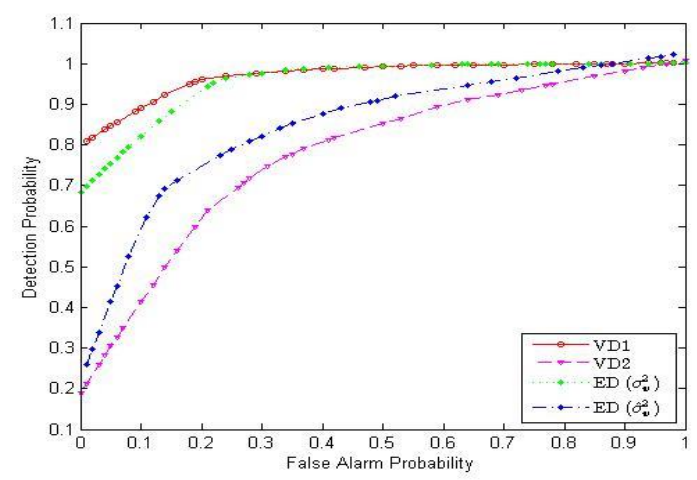

Fig. 2(b) Non-uniform noise for $\mathrm{m}=4$.

In the meantime the Fig. 2(b) represents the non-uniform noise. For this the noise power levels are $[0,1.7,-0.7,-2] \mathrm{dB}$. In the circumstance of non-uniform noise, the Energy Detector $\left(\hat{\sigma}_{w}^{2}\right)$ approach strategy fundamentally outflanks the non-robust detectors, for example Volume-based detector $2\left(V D_{2}\right)$ approach. By the by, it is substandard to the $\left(V D_{1}\right)$ calculation which is better as the benchmark, as portrayed in Fig. 2(b)

We have to say that, the Energy detector and $V D_{1}$ proportions plans are strong against the non-uniform noise, and the previous outflanks the last in precision. It ought to be brought up here in that as compared to Energy detection technique with our proposed technique $V D_{1}$ require an extra imperative that the edges of the geometry shaped by the sample covariance matrix are standardized to one. Without this limitation the volume should be increased because the edges of the geometry in the energy detector proportion approaches are bigger than one in the presence of licensed users. As a result the Energy detection method is not so significant as compared with the $V D_{1}$. So, the volume based detection performance.

The Fig. 3 represents the exact outcome of another parameter, where the signal to noise ratio is $-10 \mathrm{~dB}$, number of samples(n) is 100 and the number of antenna elements $(\mathrm{m})$ is 6 .

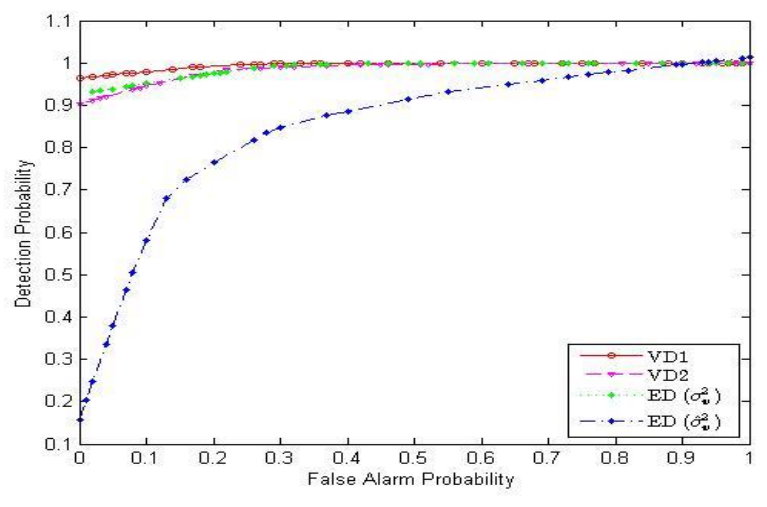

Fig. 3(a) Uniform noise for $m=6$. 


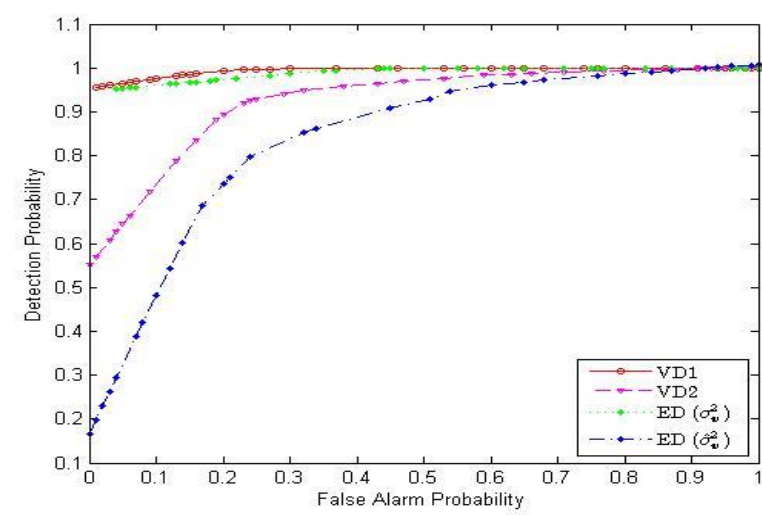

Fig. 3(b) Non-uniform noise for $\mathrm{m}=6$.

The Fig. 3(a) and Fig. 3(b) represents the uniform and non-uniform noises respectively. In uniform case the $\operatorname{ED}\left(\sigma_{w}^{2}\right)$ and $V D_{2}$ methods are very close to each other. As compared with $V D_{2}$ and $\operatorname{ED}\left(\sigma_{w}^{2}\right)$ techniques $V D_{1}$ algorithm plays out the best. By this we observe that the $\operatorname{ED}\left(\hat{\sigma}_{w}^{2}\right)$ detector does not perform well when the sample size is more. In non-uniform case, the noise power levels are $[0,-1,1.5$, $0.8,2,-1.7] \mathrm{dB}$ which is for 6 different antennas. From the fig. 3(b) we say that the $V D_{1}$ approach perform well and keep their detection accuracy but the $\operatorname{ED}\left(\hat{\sigma}_{w}^{2}\right)$ and $V D_{2}$ techniques, agonizes from performance dilapidation.

By optimizing antenna elements from $m=4$ to $m=6$ we can reduce the false alarm probability. This performance is applicable only for six antenna elements in the case of volume-Based detection. Even the number of antenna elements is increased then the performance results same as $m=6$, there is no improvement.

\section{Conclusion}

For spectrum sensing we have to propose a two novel volume based methods. By this method we reduce the multipath fading, shadowing and false alarm probabilities as compared with energy detector technique. In the casing work of Random Matrix Theory, the approximation of the determinant of the Sample Covariance Matrix, specifically, the volume, can be precisely decided, which ends up being a straight forward Gaussian distribution. By using this approximation $\gamma_{2}$ which is the theoretically threshold for $V D_{2}$ technique is precisely dictated, especially for the scenario when the values of $m$ and $n$ are huge and near each other. The outcomes of the Numerical results concur well with the theoretical values. The theoretical threshold for volume detector $1\left(V D_{1}\right)$ cannot be computed. In the meantime the hypothetical location probabilities of the $V D_{1} \& V D_{2}$ approaches cannot be figured yet. In our future works these problems will be handled.

\section{References}

[1] Lei Huang, H.C.So, Cheng Qian, Volume Based Method for Spectrum sensing, Digital signal processing 28 (2014) 48-56.

[2] B.I.Ahmad,A.Tarczynski, Reliable wide band multichannel spectrum sensing using randomized sampling schemes, Signal Process 90(7)(Jul.2010)2232- 2242.

[3] F.-X.Socheleaua, S.Houckea, P.Ciblatb,A.Aïssa-El-Beya,Cognitive OFDM system detection using pilottones secondand third order cyclostationarity, Signal Process.91(2)(Feb.2011)252-268.
[4] B.Seo,Precoder design in cognitive radio networks with channel covariance information, Signal Process.92(12)(Dec.2012)3056-3061.

[5] F.F.Digham,M.S.Alouini,M.K.Simon,On the energy detection of unknown signals over fading channels,EEETrans.Commun.55(1)(Jan.2007)21-24.

[6] B.Shen,L.Huang,C.Zhao,Z.Zhou,K.Kwak,Energy detection based spectrum sensing for cognitive radio sin noise of uncertain power, in:Proc.International Symposium on Communications and Technologies(ISCIT),Oct.2008,pp.628-633.

[7] T.J.Lim,R.Zhang,Y.-C.Liang,Y.Zeng,GLRT spectrum sensing for cognitive radio,in:Proc.IEEE Commun.Conf.(GLOBECOM), NewOrlea ns,LO,Nov.2008,pp.1-5.

[8] Y.Zeng,Y.-C.Liang, Eigen value-based spectrum sensing algorithms for cognitive radio, $\quad$ IEEE Trans. Commun.57(6)(Jun.2009)1784-1793.

[9] A.Kortun,T.Ratnarajah,M.Sellathurai,C.Zhong,C. B.P apadias,Ontheper -formance of eigen value based cooperative spectrum sensing for cognitive ratio, IEEE J.Sel.Top .Signal Process. 5(1), (Feb.2011) 4955.

[10] A.Taherpour, M.Nasiri Kenari,S.Gazor, Multiple antenna spectrum sensing in cognitive radios, IEEETrans.Wirel.Commun.9(2)(Nov.2010)814-823.

[11] B.Nadler, F.Penna, R.Garello, Performance of eigen value based signal detectors with known and unknown noise level, in:Proc.IEEE International Conference on Communications(ICC), Kyoto, Japan, Jun.2011,pp.1-5.

[12] P.Wang, J.Fang, N.Han,H.Li, Multiantenna assisted spectrum sensing for cognitive radio, Trans.Veh.Technol.59(4) (May2010)1791-1800.

[13] L.Wei, O.Tirkkonen, (May2012)1268-1277.

[14] C.A.Tracy,H.Widom, On orthogonal and simplectic matrix ensembles, Commun.Math.Phys.177(1996)7 27-754.

[15] D.Ramírez,G.Vazquez-Vilar, R.López-Valcarce, J.Vía, I.Santamaría, Detection of rank-p signals in cognitive radio networks with uncalibrated multiple antennas,IEEETrans.SignalProcess.59(8 )(Aug.2011) 3764-3774

[16] J.K.Tugnait, On multiple antenna spectrum sensing under noise variance uncertainty and flat fading, IEEE Trans. Signal Process. 60(4) (Apr.2012)1823-1832.

[17] A. Mariani, A. Giorgetti, M. Chiani, Test of independence for cooperative spectrum sensing with uncalibrated receivers, in: Proc. Of IEEE Global Communications Conference (GLOBECOM),Anaheim, CA, 3-7 Dec. 2012,pp.1374-1379.

[18] D.Ramírez, J.Vía, I.Santamaría, The locally most powerful test formulating antenna spectrum sensing with uncalibrated receivers, in:Proc.IEEEInternationa 1 Conference on Acoustics, Speech and Signal Processing(ICASSP), Kyoto,Japan,March2012,pp.3 437-3440.

[19] N.Giri, On the complex analysis of $T 2$-and $R 2$ tests,Ann.Math.Stat.36(1965)665-670.

[20] B.T.Porteous, Improved likelihood ratio statistics for covariance selection models,Biometrika72(1985) 97-101.

[21] D.Jonsson, Some limit theorems for theEigenvalues of a sample covaria nce matrix, J.Multivar.Anal.12(1982)1-38.

[22] Z.D.Bai, J.W.Silverstein, CLT for linear spectral statistics of a large dimensional sample covariance matrix, Ann.Probab.32(2004)553-605.

[23] M.D.Springer, Algebra of Random Variables, Wiley, New York, 1973.

[24] R.J.Muirhead, Aspects of Multi variate Statistical Theory, Wiley, New York, 1982.

[25] Q.T.Zhang, Advanced detection techniques for cognitive radio, in:Proc.IEEE International Conference on Communications(ICC), Dresden,Germany,Jun.2009,pp.1-5.

[26] Shaik Yasmin Fathima, Md. Zia Ur Rahman, K. Murali Krishna, Shakira Bhanu,Mirza Shafi, "Side Lobe Suppression in NC-OFDM Systems Using Variable Cancellation Basis Function", IEEE Access, vol.5, no.1, pp. 9415-9421, 2017.

[27] Q.T.Zhang, Theoretical performance and threshold of the multi taper method for Trans.Veh.Technol. 60(5) (Jun.2011)2128-2138 spectrum sensing, IEEE 